"This document is the Accepted Manuscript version of a Published Work that appeared in final form in ACS Sensors, copyright @ American Chemical Society after peer review and technical editing by the publisher. To access the final edited and published work see https://pubs.acs.org/doi/10.1021/acssensors.8b00477 


\section{Advanced Photonic Sensors Based on Interband Cascade Lasers for Real-Time Mouse Breath Analysis}

Erhan Tütüncü, ${ }^{\dagger}$ Markus Nägele, ${ }^{\ddagger}$ Steffen Becker ${ }^{\S}$ Marc Fischer ${ }^{\S}$ Johannes Koeth, ${ }^{\S}$ Christian Wolf, Stefan Köstler, ${ }^{\|}$Volker Ribitsch, ${ }^{\perp}$ Andrea Teuber, ${ }^{\dagger}$ Michael Gröger, ${ }^{\#}$ Sandra Kress, ${ }^{\#}$ Martin Wepler, Ulrich Wachter, Josef Vogt, ${ }^{\#}$ Peter Radermacher, ${ }^{\#}$ and Boris Mizaikoff*, ${ }^{\dagger}$

${ }^{\dagger}$ Institute of Analytical and Bioanalytical Chemistry, Ulm University, Albert-Einstein-Allee 11, 89081 Ulm, Germany

‡OptoPrecision GmbH, Auf der Höhe 15, 28357 Bremen, Germany

${ }^{\S}$ nanoplus Nanosystems and Technologies GmbH, Oberer Kirschberg 4, 97218 Gerbrunn, Germany

"Joanneum Research Forschungsgesellschaft mbH., Materials - Institut für Oberflächentechnologien und Photonik,

Franz-Pichler-Straße 30, 8160 Weiz, Austria

${ }^{\perp}$ TecSense GmbH, Teslastraße 4, 8074 Grambach, Austria

\#Institute of Anesthesiologic Pathophysiology and Method Development, Ulm University Medical Center, Helmholtzstr. 8/1, 89081 Ulm, Germany

\section{Supporting Information}

ABSTRACT: A multiparameter gas sensor based on distributed feedback interband cascade lasers emitting at $4.35 \mu \mathrm{m}$ and ultrafast electro-spun luminescence oxygen sensors has been developed for the quantification and continuous monitoring of ${ }^{13} \mathrm{CO}_{2} /{ }^{12} \mathrm{CO}_{2}$ isotopic ratio changes and oxygen in exhaled mouse breath samples. Mid-infrared absorption spectra for quantitatively monitoring the enrichment of ${ }^{13} \mathrm{CO}_{2}$ levels were recorded in a miniaturized dual-channel substrate-integrated hollow waveguide using balanced ratiometric detection, whereas luminescence quenching was used for synchronously detecting exhaled oxygen levels. Allan variance analysis verified a $\mathrm{CO}_{2}$ measurement precision of $1.6 \%$ during a $480 \mathrm{~s}$ integration time. Routine online monitoring of exhaled mouse breath was performed in 14 mechanically ventilated and instrumented mice and demonstrated

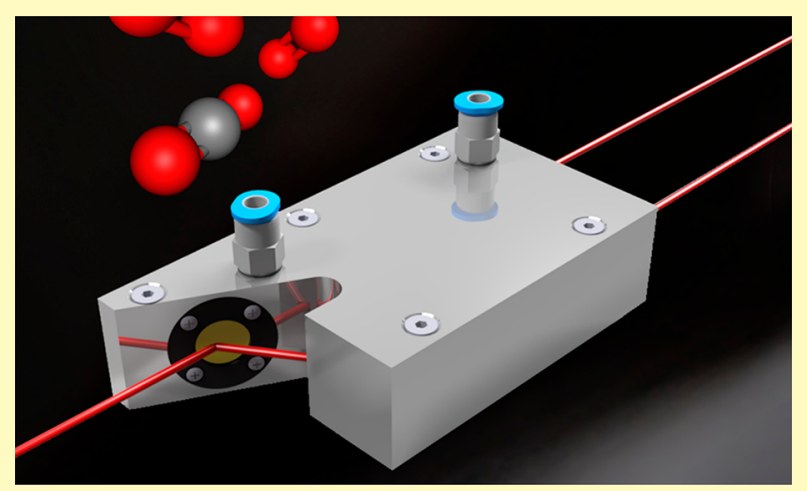
the feasibility of online isotope-selective exhaled breath analysis within microliters of probed gas samples using the reported combined sensor platform.

KEYWORDS: substrate integrated hollow waveguides, breath analysis, interband cascade laser, electrospun polymer nanofiber, isotope enrichment

$\mathrm{E}$ xhaled breath analysis (EBA) has attracted a considerable amount of scientific and clinical interest, as noninvasive real-time identification and quantification of potential disease biomarkers offers substantial clinical potential. Due to the low concentrations of biomarkers, highly selective and sensitive analytical instruments and methods are required for the detection and quantification of relevant biomarkers for disease diagnostics and metabolic status monitoring. The latter is based on the analysis of endogenously produced breath compounds or the analysis of breath metabolites after substrate administration. Established biomarkers such as exhaled carbon dioxide $\left(\mathrm{CO}_{2}\right)$ and the carbon isotopologues, ${ }^{13} \mathrm{CO}_{2}$ and ${ }^{12} \mathrm{CO}_{2}$, have been successfully demonstrated for the diagnosis of Helicobacter pyroli infections, liver malfunction, bacterial overgrowth, and fat absorption. ${ }^{1,2}$ Changes in isotopic ratio of both isotopes is herein observed by administration of ${ }^{13} \mathrm{C}$ - enriched sugars and may directly be assigned to alterations of metabolization processes. The increase of the isotope ratio in the expired breath (a.k.a., enrichment) allows one to noninvasively monitor glucose metabolism. Analytical techniques meeting the requirements of high precision and accuracy for breath gas analysis include gas chromatography (GCMS $)^{3,4}$ and isotope ratio mass spectrometry (IRMS). ${ }^{5}$ Although these techniques demonstrate excellent figures-ofmerit for $\delta^{13} \mathrm{C}$ analysis, their routine establishment is limited due to large instrumental dimensions. Moreover, continuous online measurements next to mechanically ventilated animals are not feasible, as sampling is required. Online monitoring

Received: June 8, 2018

Accepted: August 3, 2018

Published: August 3, 2018 
capabilities for exhaled breath analysis will only gain momentum if near real-time optical/spectroscopic techniques are harnessed for the determination of relevant exhaled breath biomarkers in microliter sample volumes, as required in mouse breath analysis.

Spectroscopic techniques for online monitoring and quantification of breath components have recently attracted attention due to continuous advancements in semiconductor lasers technology. High sensitivity and selectivity for trace concentration detection of breath biomarkers has taken advantage of tunable diode laser absorption spectroscopy (TDLAS), photoacoustic spectroscopy, ${ }^{6}$ and cavity ring-down spectroscopy. ${ }^{7}$ Optical methods based on infrared laser spectroscopy for $\mathrm{CO}_{2}$ and according isotope pairs exploit the pronounced fundamental vibrational band $\nu_{3}$ of $\mathrm{CO}_{2}$ in the mid-infrared (MIR) spectral range at $4.3 \mu \mathrm{m}$. With advancements in diode laser technology, and thus increasing availability of light sources emitting in the 3-6 $\mu \mathrm{m}$ regime at reasonable output power, interband cascade lasers (ICL) $)^{8-12}$ are of particular interest. ICLs offer emission in the wavelength regime of $3-6 \mu \mathrm{m}$ at room temperature, provide the required wavelength tunability, and feature low power consumption, as needed for future portable devices. In addition to the determination of the isotope ratio in low-volume samples via the developed sensing system, fundamental insight into the metabolic status of the mechanically ventilated small animals is offered via calculation of the respiratory quotient (RQ). Significant advances in sensor response time of optochemical sensors have been achieved by processing the utilized polymer into nanofibers as introduced by Köstler and collaborators. ${ }^{13}$ Highly porous nanofiber structures were prepared by electrospinning the developed polymer formulation of PtTFPP (platinum(II)-5,10,15,20-tetrakis(2,3,4,5,6-pentafluorphenyl)porphyrin) immobilized in polystyrene.

In this article, we describe the development of a compact multiparameter sensor system based on a combination of TDLAS and optical oxygen sensors for online and continuous quantification of the ${ }^{13} \mathrm{CO}_{2} /{ }^{12} \mathrm{CO}_{2}$ isotope ratio and oxygen and, subsequently, determination of the respiratory quotient in mechanically ventilated and surgically instrumented mice. The obtained results were compared to GC-MS measurements for validation.

\section{RESULTS AND DISCUSSION}

The performance of the developed breath sensor system was evaluated in terms of sensitivity, precision, and linearity. Figure 1a illustrates experimental direct absorption signal of both transitions, $\mathrm{P}(58)$ and $\mathrm{R}(14)$, respectively, for the ${ }^{12} \mathrm{C}$ and the ${ }^{13} \mathrm{C}$ isotopes around $2294 \mathrm{~cm}^{-1}$. The spectrum is obtained from a real mouse breath sample showing clearly enriched ${ }^{13} \mathrm{CO}_{2}$ concentrations.

As shown in Figure 1a, the selected spectral window around $2294 \mathrm{~cm}^{-1}$ offers resolved absorption lines of ${ }^{12} \mathrm{CO}_{2} \mathrm{P}(58)$ and ${ }^{13} \mathrm{CO}_{2} \mathrm{R}(14)$ suitable for data analysis. The intensity ratio of both isotope lines has been calculated as 1.16/1.00 (based on line strengths derived from the HITRAN database ${ }^{14}$ for the ratio of natural abundance $\left({ }^{13} \mathrm{CO}_{2} /{ }^{12} \mathrm{CO}_{2}: 1.11 / 98.89\right)$. Further absorption lines (P $46 \mathrm{f} 2294.34 \mathrm{~cm}^{-1}$, P47e 2293.38 $\mathrm{cm}^{-1}$ ) are expected in this spectral region but, however, could not be resolved due to chirp-limited spectral resolution and rather broad absorption features at atmospheric pressure. As a consequence, a surface response fit was used for quantitative
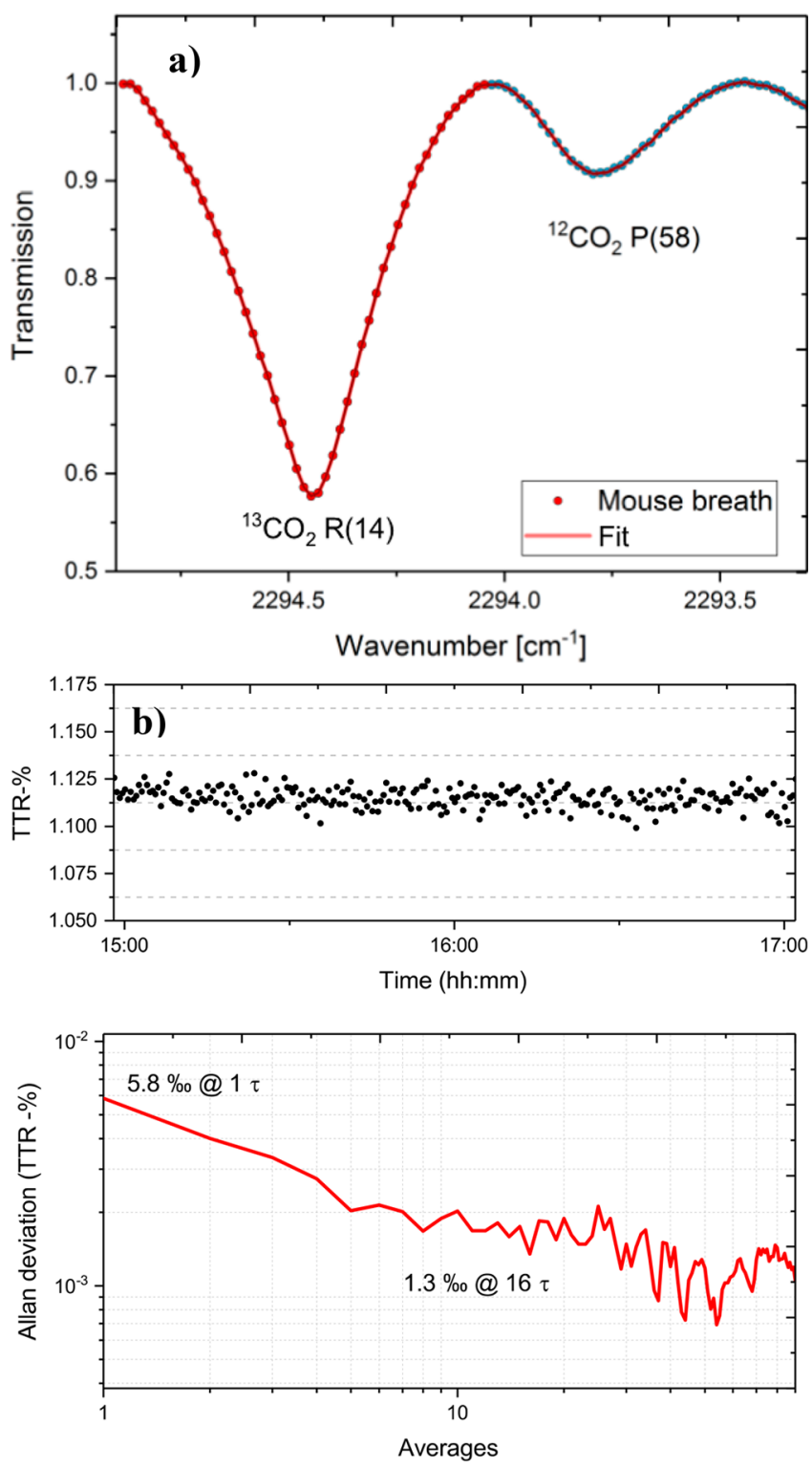

Figure 1. (a) Experimentally determined transmission spectrum of a mouse breath sample at $303 \mathrm{~K}$ and 980 mbar. (b) Allan variance analysis revealing a precision of $1.3 \%$ when averaging 16 data points.

analysis of both isotopes in order to compensate for any occurring influence by underlying lines. Hence, despite the fact that underlying $\mathrm{CO}_{2}$ absorption lines are not resolved herein, their influence was compensated with sufficient accuracy.

The $\delta$ value precision was evaluated via an Allan-Werle deviation analysis. ${ }^{15,16}$ The TDL was operated at a sample flow rate of $20 \mathrm{~mL} \mathrm{~min} \mathrm{mi}^{-1}$ and a sampling interval of 2 measurements $/ \mathrm{min}$. As illustrated in the Allan deviation plot in Figure 1b, the developed isotopic ratio sensor achieved a precision of $\sim 1.3 \%$ when averaging 16 measurements (i.e., over a period of $8 \mathrm{~min}$ ) for the TTR-\%. Measurements of the $\mathrm{CO}_{2}$ mole fraction $\left({ }^{13} \mathrm{CO}_{2}\right.$ and $\left.{ }^{12} \mathrm{CO}_{2}\right)$ were made using certified gas standards of $3 \mathrm{vol} \%$ total $\mathrm{CO}_{2}$ in nitrogen for $5 \mathrm{~h}$, indicating a TDLAS intra-assay precision of $\pm 4.8 \%$ for total $\mathrm{CO}_{2}$. As the TDLAS system is meant to be used during extended measurement periods (i.e., on average $8 \mathrm{~h}$ ), the drift behavior was tested during a $16 \mathrm{~h}$ measurement of $3 \% \mathrm{CO}_{2}$ (intra-assay precision of $8.85 \% 0$ ), and drift-free operation was 
demonstrated. The obtained precision level is comparable to complementary approaches using laser spectroscopy for detecting isotope ratios, which operate at reduced sample gas pressures to achieve sufficient spectral resolution. The present approach offers real-time analysis of mouse breath at atmospheric pressure; therefore, vacuum equipment is not needed. Furthermore, the developed double-channel substrate integrated hollow waveguide (dciHWG) offers the ability to simultaneously record sample and reference signals in a balanced detection scheme to obtain the difference in $\delta$, that is, using the reference channel for the secondary isotope standard.

Comparison TDLAS versus MS. The performance of the senor was tested for the determination of the TTR ratio (i.e., tracer-to-tracee ratio $=\left({ }^{13} \mathrm{CO}_{2} /{ }^{12} \mathrm{CO}_{2}\right) \times 100 \%-$ basal ${ }^{13} \mathrm{CO}_{2}$ abundance in exhaled breath) and for total $\mathrm{CO}_{2}$ calculated via the ${ }^{13} \mathrm{CO}_{2}$ and ${ }^{12} \mathrm{CO}_{2}$ concentrations within real exhaled mouse breath samples and comparing the values validated via GC/MS studies performed using the same mouse breath samples. For glucose metabolism monitoring, ${ }^{13} \mathrm{C}$-enriched glucose was administered during the studies presented herein, resulting in exhalation of ${ }^{13} \mathrm{CO}_{2}$. The variation in exhaled tracer levels was monitored over an $8 \mathrm{~h}$ test duration, where breath samples were collected hourly for reference GC/MS measurements. Initial laboratory experiments indicated that the observed TDLAS versus MS offset was caused by pressure broadening due to $\mathrm{N}_{2}$ serving as the background gas. This offset was avoided by calibrating the TDLAS system with $\mathrm{CO}_{2}$ mixed with synthetic air $\left(\mathrm{O}_{2} 20 \%, \mathrm{~N}_{2} 80 \%\right)$. Finally, the developed sensor system was deployed for online breath analysis during 14 mouse breath studies.

The obtained results for $\mathrm{CO}_{2}$ and TTR determination are shown in Figure 2a,b. The orthonormal regression analysis yields a slope of $\mathrm{CO}_{2 \mathrm{GC}-\mathrm{MS} / \mathrm{TDLAS}}=0.987 \pm 0.008\left(R^{2}=\right.$ $0.995)$ and TTR-\% GC-MS/TDLAS $=0.994 \pm 0.004\left(R^{2}=0.995\right)$, corroborating excellent agreement between the developed TDLAS setup and GC/MS validation measurements. A mean deviation for the TTR of 12 and $14 \%$ of for $\mathrm{CO}_{2}$ determination was obtained. The concentration of exhaled $\mathrm{CO}_{2}$ ranged from 2.36 to $3.97 \%$, and the obtained TTR values ranged from 0.78 to $7.95 \%$ (112 measurements executed in total). Exemplary kinetic profiles obtained for four mice are shown in the Supplementary Figure S- 9. All profiles show a fast increase in enrichment after administration of ${ }^{13} \mathrm{C}$-enriched sugar. Depending on the individual glucose metabolism of the surgically instrumented mice, the TTR values decrease over time.

The comparison shows that the developed sensor system provides high accuracy during continuous monitoring of ${ }^{13} \mathrm{CO}_{2}$ to ${ }^{12} \mathrm{CO}_{2}$ ratios and for total $\mathrm{CO}_{2}$ in exhaled mouse breath without the need of sampling, sample storage, and timedelayed analysis as required by GC/MS. Moreover, the application of TDLAS enables obtaining real-time concentration values throughout the duration of the test, which could not be obtained by any other method. First approaches toward mouse breath analysis in the MICU were demonstrated by our research team based on Fourier transform infrared spectroscopy approaches using sophisticated chemometric strategies requiring advanced data treatment and complex calibration procedures. $^{17-19}$ Due to accurate and robust optics and electronics in combination with integrative fitting algorithms, the TDLAS system uniquely facilitates online signal processing and evaluation.
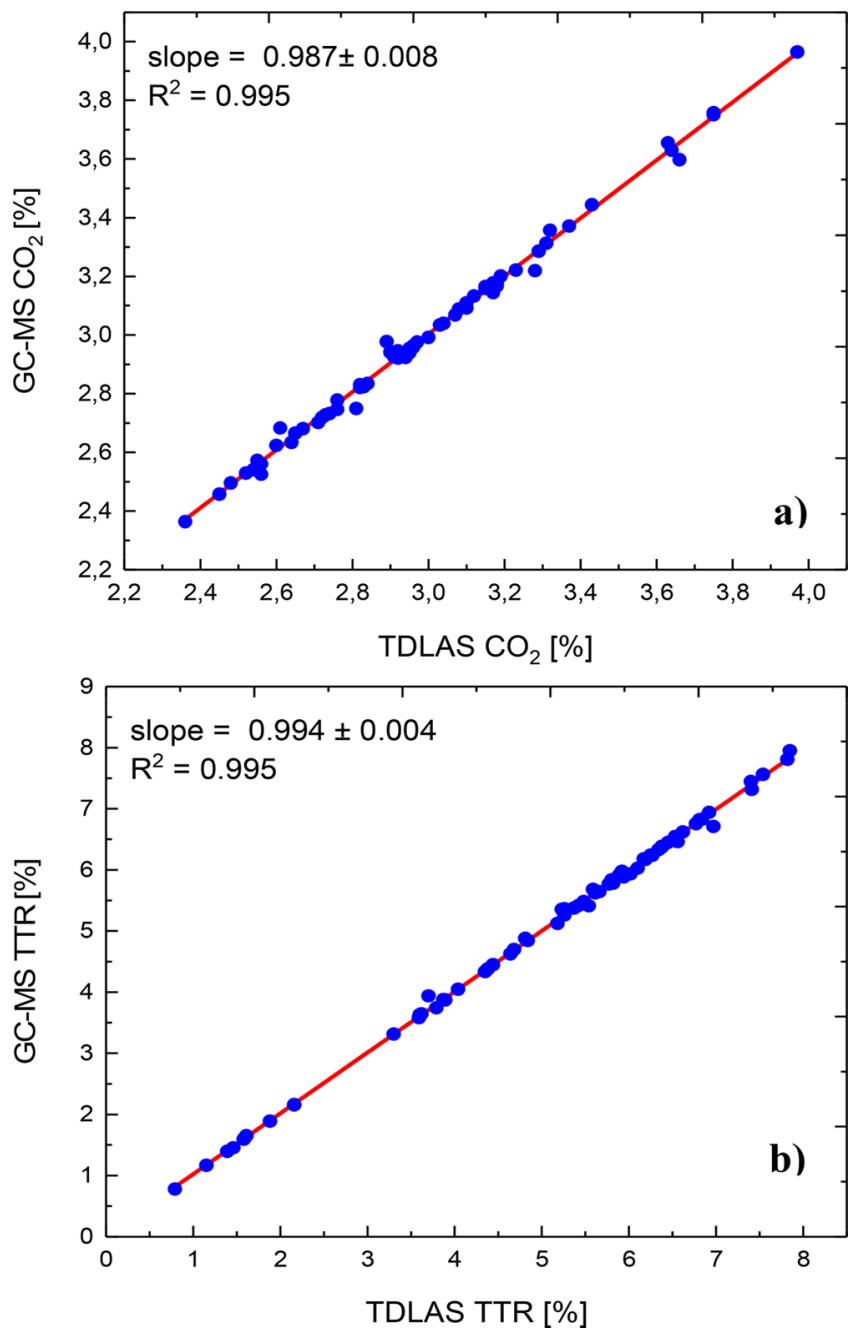

Figure 2. Correlation between dciHWG-TDLAS and GC/MS measurements of (a) $\mathrm{CO}_{2}$ and (b) TTR-\% of exemplary exhaled mouse breath samples.

Oxygen Sensing. In addition to the analysis of the isotope ratio, the developed sensor system is also capable of monitoring the respiratory quotient with additional oxygen sensing via the integrated optochemical polymer nanofiber sensor. As previously described, two absorption spectra and 12 oxygen measurement points were recorded every minute.

The calculation of the respiratory quotient in exhaled mouse breath was based on measuring the ratio of the volume of exhaled carbon dioxide $\left(V_{c}\right)$ to the volume of oxygen consumed $\left(V_{\mathrm{o}}\right)$. The precision and long-term stability of the oxygen sensor were analyzed. The oxygen sensor shows an average minimum 99\% recovery after being purged with nitrogen, indicating high reversibility of the sensor. Relative standard deviations are in the range of $0.14 \%$ for oxygen-free medium to $0.19 \%$ o for a $20 \%$ oxygen mixture, thus revealing the capability of high-precision oxygen measurements. Longterm stability of the utilized electrospun oxygen sensor was tested by the continuous determination of the phase shift of a $20 \%$ oxygen mixture in nitrogen for $16 \mathrm{~h}$. Every $5 \mathrm{~s}$, a phase shift was determined and directly converted into an oxygen concentration. A relative standard deviation of $0.39 \%$ was found for a $16 \mathrm{~h}$ measurement period at a flow rate of $20 \mathrm{~mL} /$ min. No discernible drift of the signal was revealed, although permanent illumination is known to cause photoinduced dye 
oxidation and photobleaching. The limit of detection (LOD) of the oxygen sensor was calculated as LOD $=(3.3 \sigma / k)$, where the standard deviation $(\sigma)$ is the standard deviation of the $y$ intercepts of regression and $k$ is the slope of the calibration graph. The LOD was determined at approximately $0.003 \%$ oxygen, indicating a sufficiently high sensitivity. The system response time was determined to be $10 \mathrm{~s}$ for a flow rate of 20 $\mathrm{mL} / \mathrm{min}$, resulting from gas exchange within the tubing volume. Increasing the flow rate of the pump to a flow rate of $200 \mathrm{~mL} / \mathrm{min}$ led to a rise time of $<2 \mathrm{~s}$, indicating a higher gas exchange rate. It should be noted that the slow flow rate for system testing was selected to correlate with real mouse breathing periods $(15-20 \mathrm{~mL} / \mathrm{min})$.

Monitoring of the Respiratory Quotient. The developed sensor system clearly provides sufficient sensitivity and time resolution for enabling real-time exhaled $\mathrm{CO}_{2}$ and $\mathrm{O}_{2}$ monitoring, as shown in Figure 3. Conventional capnography monitors and blood gas analyzers have only limited applicability in mechanically ventilated mice for characterization of the energy metabolism characterization, due to the low blood volume and small tidal volume.

To calculate the RQ in mouse breath, the difference in exhaled and inhaled oxygen $\left(\Delta \mathrm{O}_{2}\right)$ is calculated by monitoring
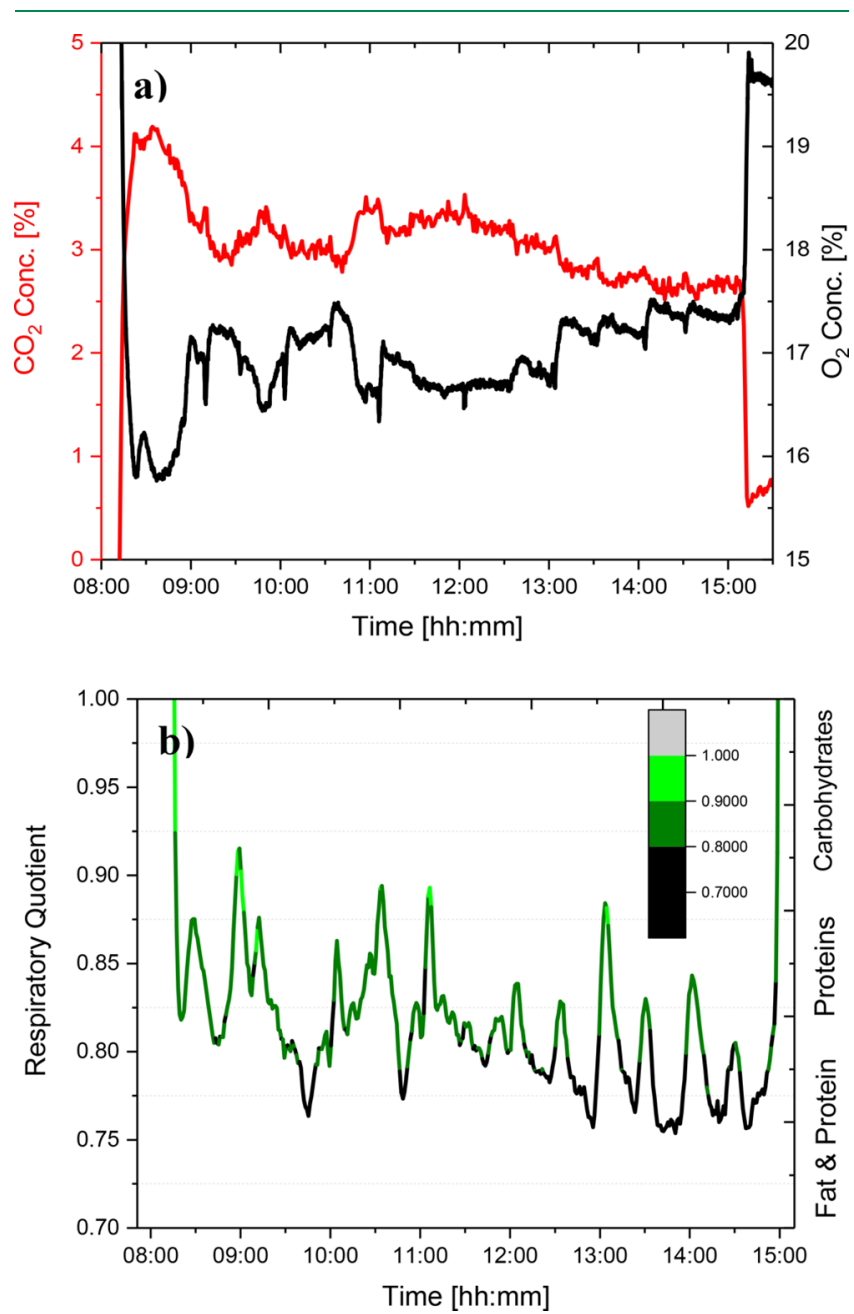

Figure 3. (a) Example of continuous monitoring of exhaled carbon dioxide (red) and oxygen (black) from a ventilated mouse. (b) Respiration quotient as a function of time during an exemplary mouse experiment. the oxygen content of both inhaled and exhaled air. Thus, long-term monitoring of the oxygen content in compressed air used for mechanical ventilation was performed, resulting in an average oxygen concentration of $20.77 \pm 0.05 \%$. Furthermore, precise synchronization of the two senor signals-IR and luminescence-is essential. The implemented signal processing routine automatically determines the inhaled and exhaled oxygen concentration and the exhaled carbon dioxide concentration, which enables direct monitoring of the respiratory quotient in exhaled mouse breath almost in real time. In Figure $3 b$, an exemplary trajectory of the respiratory quotient response of a mouse experiment starting at 08:00 and ending at approximately 14:45 is shown.

A time delay of approximately $5 \mathrm{~min}$ between breath exhalation and signal generation was inevitable due to the required tubing for gas transportation, resulting from the rather slow respiratory flow from the mouse. All connection tubing was kept as short as possible to minimize the dead volume and reduce the delay in data acquisition. Prominent peaks and dips due to intermittent changes in ventilation settings and fluctuations were smoothed due to the gas reservoir between the expiratory branch of the respiratory unit and the breath analyzer. Using additional smoothing functions in the data evaluation procedure, that is, short moving average (3 data points) in combination with Savitzky-Golay filter, provided smoothed response data without losing any essential information. Data points in Figure 3 are shown every $60 \mathrm{~s}$, resulting in sufficient temporal resolution for facilitating physiologically meaningful respiratory data interpretation.

Indicating a metabolic shift from carbohydrate metabolism to fat oxidation with increasing duration of the mouse experiment, the determination of RQ values of all 14 mouse experiments executed so far revealed a common trend with respect to the decrease in $R Q$ value toward the end of the protocol to approximately 0.75 . In addition to the significant drop of RQ values over time, sharp increases and declines are evident in intervals of $30 \mathrm{~min}$. These peaks are caused by socalled recruitment maneuvers (RMs). ${ }^{20} \mathrm{RMs}$ are performed to reopen/re-expand collapsed lung tissue occurring by inflation of lung subunits. The usefulness of recurrent RMs in mechanically ventilated mice with the specific aim to maintain lung mechanics and other lung functions has been explored by Reiss et al. ${ }^{21}$ It could be shown that RMs reduce the development of pulmonary inflammation and prevent atelectasis (i.e., incomplete expansion of the lung).

In summary, the successful combination of a low-volume dual-channel iHWG with ICL-based TDLAS and complementary yet synchronized luminescence oxygen sensors enabled the detection of various relevant biomarker molecules serving as an advanced diagnostic strategy in exhaled mouse breath analysis. To the best of our knowledge, this is the first report of a real-time multispectral gas sensor system enabling continuous metabolic status monitoring via in-line analysis of minute mouse breath samples during medical trials with a time resolution at the scale of seconds. The innovative dciHWG gas cell offers rapid gas exchange while simultaneously serving as a photon conduit and is designed to support a balanced detection scheme for efficiently eliminating noise. In a next step, expanding the capabilities of this measurement technique via appropriate preconcentration schemes and extending the IR wavelength regime to address further trace biomarkers via their vibrational fingerprint is anticipated. 


\section{CONCLUSION}

An innovative all-optical sensor system combining MIR interband cascade laser technology with miniaturized dualchannel substrate-integrated hollow waveguide gas cells and nanofiber-based luminescence schemes enables online monitoring of important metabolic parameters, including total $\mathrm{CO}_{2},{ }^{13} \mathrm{CO}_{2}$ isotope enrichment, and $\mathrm{O}_{2}$ in exhaled breath of mechanically ventilated mice. An ICL tunable diode around $4.35 \mu \mathrm{m}$ was used for time-resolved infrared absorption spectroscopy to determine ${ }^{12} \mathrm{CO}_{2}$ (P58) and ${ }^{13} \mathrm{CO}_{2}$ (R14) during the respiration of mice in a mouse intensive care unit. The breath analyzer was integrated into the respiratory equipment of the so-called "mouse intensive care unit" (MICU) and enables real-time direct exhaled breath monitoring in microliter sample volumes. In combination with the integrated oxygen sensor, accurate determination of the basal metabolic rate based on online access to the respiratory quotient is facilitated. Excellent agreement of the obtained results was confirmed by validation via GC/MS. The developed breath analysis system is now in routine use in the MICU at the Institute of Anesthesiologic Pathophysiology and Process Engineering at the Ulm University Medical Center. The presented sensor is anticipated to facilitate online metabolic status monitoring in small animal models with the potential to be scaled for alternative sensing scenarios where addressing minute volumes of probed gas is challenging.

\section{EXPERIMENTAL SECTION}

Sensor Configuration. In Figure 4a, the developed portable sensing system comprising the TDLAS system for determination of ${ }^{13} \mathrm{CO}_{2} /{ }^{12} \mathrm{CO}_{2}$ ratio and the optical oxygen sensor is illustrated. The combined sensor architecture is packaged into a standard 19 in. housing with dimensions of $471 \times 451 \times 192 \mathrm{~mm}(L \times W \times H)$. In situ online monitoring of exhaled breath components was achieved by locating the senor system in-line within the expiratory branch. A small animal respiratory system/ventilator was used for stable ventilation of treated mice. A $100 \mathrm{~mL}$ gas reservoir was placed between the expiratory branch and the sensor system to stabilize breath humidity and to reduce fluctuations.

For isotopic measurements, a thermoelectrically cooled distributed feedback (DFB) interband cascade laser (S/N: 2082/04-09, nanoplus $\mathrm{GmbH}$ ), emitting single mode infrared radiation at 2294 $\mathrm{cm}^{-1}(4.35 \mu \mathrm{m})$, was used. Further details on the ICL characteristics are provided in the Supporting Information. The laser diode was packaged in TO-66 housing and was equipped with a collimating lens. In the MIR spectral region, pronounced fundamental absorption features of $\mathrm{CO}_{2}$ are located at $4.3 \mu \mathrm{m}$, which are 100 times stronger than the $\nu_{2}$ band centered at $1.5 \mu \mathrm{m}$ and the combination band $\nu_{1}+$ $\nu_{3}$ centered at $2.7 \mu \mathrm{m}$. Several line pairs characteristic for ${ }^{13} \mathrm{CO}_{2} /{ }^{12} \mathrm{CO}_{2}$ can be addressed for direct absorption spectroscopy. High-precision isotopic ratio measurements for mouse breath analysis are accomplished by selecting absorption features with almost equal line strength. In order to prevent spectral interference of both isotopes, the respective absorption lines must be well-separated for accurate data analysis, yet need to be located within the tuning range of a single ICL.

Thus, absorption lines at 2293.81 and $2294.48 \mathrm{~cm}^{-1}$ (4.3595 and $4.3582 \mu \mathrm{m})$ of ${ }^{12} \mathrm{CO}_{2}$ and ${ }^{13} \mathrm{CO}_{2}$ were selected, as these lines are sufficiently close in frequency, yet spectrally separated for line fitting. Furthermore, their absorption strength is almost equal $\left({ }^{12} \mathrm{CO}_{2}=\right.$ $3.055 \times 10^{-20} ;{ }^{13} \mathrm{CO}_{2}=3.547 \times 10^{-20}$ ) despite the disparity in relative concentrations of each isotope. It is clearly evident that the applied ICL readily covers the overlap of the P-branch of ${ }^{12} \mathrm{CO}_{2}$ and the R-branch of ${ }^{13} \mathrm{CO}_{2}$ of the $(0,0,1)-(0,0,0)$ vibrational transition in this wavelength regime.
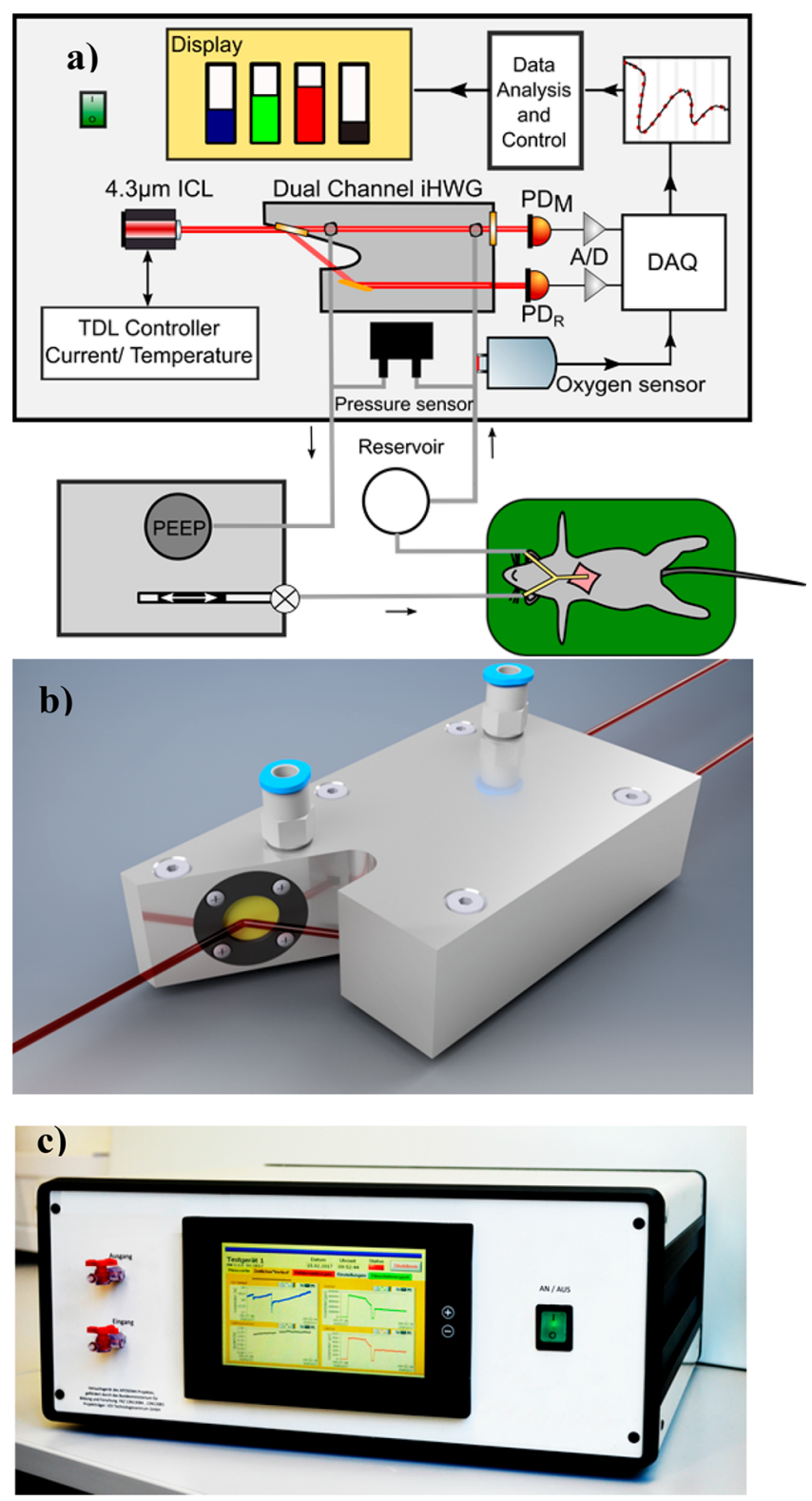

Figure 4. (a) Mouse breath experimental apparatus: PEEP, peep positive end expiratory pressure system; ICL, interband cascade laser; $\mathrm{PD}$, pyroelectric detector (measurement and reference); $\mathrm{A} / \mathrm{D}, \mathrm{AD}$ converter; DAQ data acquisition system. (b) Dual-channel substrateintegrated hollow waveguide. (c) Front view of the developed prototype mouse breath analyzer system.

Laser diode modulation was performed via a custom current/ temperature controller developed by OptoPrecision (OptoPrecision $\mathrm{GmbH}$, Bremen, Germany), enabling highly reproducible scanning across absorption lines. Wavelength tuning of the DFB ICL was performed by temperature tuning from 25.6 to $31.5^{\circ} \mathrm{C}$ at a repetition frequency of $f=4 \mathrm{MHz}$ at a pulse width of $100 \mathrm{~ns}$. A modulation frequency of $555 \mathrm{~Hz}$ was selected to ensure an optimized signal-tonoise ratio. Scanning via temperature was executed during the first $5 \mathrm{~s}$ of each measurement, and the data evaluation was carried out in the following $25 \mathrm{~s}$.

Collimated radiation from the ICL was directly coupled onto a wedged $\mathrm{ZnSe}$ window, which was integrated into the dual-channel substrate-integrated hollow waveguide serving as 50/50 beam splitter and as window of the hollow waveguide gas cell. Thus, $50 \%$ of the emitted radiation was used as a reference beam (see Figure $4 \mathrm{~b}$ ). Both beams propagate through the dciHWG gas cell $(94 \mathrm{~mm} \times 50 \mathrm{~mm} \times$ 
$22 \mathrm{~mm}, L \times W \times H)$, providing a signal channel/cell and a reference channel/cell enabling a balanced ratiometric detection for maximizing the signal stability. Hence, absorption signal and reference signal are simultaneously recorded, eliminating common mode noise. The actually sampled gas volume is probed by the IR beam propagating via the measurement channel with a $7.5 \mathrm{~cm}$ optical path length at a sample volume of $315 \mu \mathrm{L}$ achieved by a channel cross section of 2.1 $\mathrm{mm} \times 2.0 \mathrm{~mm}$. As ambient air was selected as the reference gas, the reference channel was not sealed at both ends. The open reference channel includes a gold mirror element at the bent $(L \times W \times H: 5.01$ $\mathrm{mm} \times 5.01 \mathrm{~mm} \times 0.7 \mathrm{~mm}$ ) to facilitate radiation propagation within the curved reference channel. After exiting the cell, the signal and reference beam were directly transmitted onto two synchronized pyroelectric infrared detectors (InfraTec GmbH, Dresden, Germany). In order to provide stable measurement conditions and reduce possible $\mathrm{CO}_{2}$ contribution from surrounding air, the inside of the housing (see Figure 4c) was flushed continuously with compressed air.

Sample temperature variations have a strong influence on the precision of the measurement, as the line strengths of the isotopes are affected by temperature. Thus, temperature fluctuations in sample and reference channels have to be minimized by thermostatically controlling the sample compartment of the analyzer to a constant temperature of $31.0^{\circ} \mathrm{C}$ with a stability of $\pm 0.002{ }^{\circ} \mathrm{C}$. In addition, the pressure inside the tubing system is monitored with an absolute pressure sensor (AMSYS 4711-1200, AMSYS GmbH \& Co. KG, Mainz, Germany) during all experiments. Pressure broadening was corrected by applying a correction factor using measured values of the total pressure.

For oxygen sensing, mouse breath was passed through a smallvolume optochemical temperature-stabilized flow-through cell with an integrated oxygen sensor layer. Using a green light-emitting diode (NSPES90S, Nichia) with an emission maximum of $518 \mathrm{~nm}$, the dye embedded within the polymer nanofibers was excited to luminescence, and the phase shift after quenching via molecular oxygen was detected using a photodiode. Analyzing fluorescence lifetime provides an evaluation method that is insensitive to intensity fluctuations of the light source and the fluorophore concentration, thereby also minimizing artifacts due to, for example, heterogeneously distributed fluorophores. Even more advanced, by excitation of the fluorophore using sinusoidally modulated light, the luminescence lifetime may be evaluated as the phase shift between excitation and luminescence light, which is a parameter that-from an electronic measurement point of view-may be determined with utmost robustness and precision. In principle, the emission of fluorescence is delayed versus the excitation depending on the lifetime of the excited state of the molecule. The phase shift $\varphi$ described as $\tan \phi=\omega \tau$ gives the angular frequency of the sinusoidal modulation. This phase shift may then be converted into oxygen concentration units based on the SternVolmer theory.

For the analyzer shown in Figure 4c, signal and reference outputs of both pyroelectric detectors were acquired and processed with a data acquisition system calculating the absorbance, automatically fitting the absorption lines to Gaussian line profiles, and, thus, extracting the isotopic ratio of ${ }^{13} \mathrm{CO}_{2} /{ }^{12} \mathrm{CO}_{2}$. Using the concentration information provided by the oxygen sensor then enabled RQ and the basal metabolic rate to be directly processed.

Animal Study Protocol. The study protocol applied herein was approved by the University Animal Care Committee and the Federal Authorities for Animal Research of the Regierungspräsidium Tübingen, (approved animal experimentation number: 1282). After placement of the animal on the procedure bench equipped with a heating pad and a lamp, a rectal temperature probe was inserted. In order to expose the trachea, the right internal jugular vein, and the right carotid artery, the anterior neck was incised. The lungs were mechanically ventilated with a pressure-controlled, lung-protective ventilation strategy using a small animal ventilator (FlexiVentTM, Scireq, Montreal, Canada) after intubation of the trachea. ${ }^{22}$ After a lung recruitment maneuver, respirator settings were $0.21 \mathrm{FiO}_{2}, 6-8$ $\mu \mathrm{L} \cdot \mathrm{g}^{-1}$ tidal volume (titrated to maintain arterial $\mathrm{PCO}_{2}$ at $30-40$
$\mathrm{mmHg}$ ), 150 breaths $\cdot \mathrm{min}^{-1}$ respiratory rate, $1: 2$ inspiratory/ expiratory time ratio, and $3 \mathrm{~cm} \mathrm{H}_{2} \mathrm{O}$ PEEP. Catheters were inserted into the jugular vein, the carotid artery, and the bladder. Anesthesia was maintained with continuous i.v. ketamine, fentanyl, and midazolam, titrated to reach deep sedation and analgesia as documented by complete tolerance against noxious stimuli. Normotensive hemodynamics (i.e., mean arterial pressure $>55$ $\mathrm{mmHg}$ ) were achieved by i.v. hydroxyethyl starch (maximum infusion rate $20 \mu \mathrm{L} \cdot \mathrm{g}^{-1} \cdot \mathrm{h}^{-1}$ ) in a balanced electrolyte solution (Tetraspan $6 \%$, Braun, Melsungen, Germany) and, if needed, together with continuous i.v. norepinephrine. Animals were randomly assigned to injection of vehicle $(0.9 \%$ saline $)$ or PBS $\left(5 \mu \mathrm{L} \cdot \mathrm{g}^{-1} \mathrm{~kg}^{-1}\right)$ i.p. Infused glucose $(50 \%)$ was given as stable, nonradioactive-labeled ${ }^{1,2,3,4,5,6}-\mathrm{C}_{6^{-}}$ glucose. After $\sim 8 \mathrm{~h}$, the animals were killed through blood withdrawal via the vena cava inferior. Over a total analysis period of $7 \mathrm{~h}$, each hour, two $1 \mathrm{~mL}$ samples were collected via appropriate plastic syringes for validation by GC-MS analysis. To determine both expiratory $\mathrm{CO}_{2}$ concentration and $\mathrm{CO}_{2}$ tracer enrichment, the masses of $\mathrm{m} / z 44$ and $\mathrm{m} / z 45$ were analyzed and evaluated. $\mathrm{CO}_{2}$ production rates were calculated as the product of tidal volume, respiratory rate, and $\mathrm{CO}_{2}$ concentration. For online mouse breath analysis via the developed TDLAS system, the analyzer was integrated into the respiratory equipment of the anesthetized mouse, enabling continuous online monitoring. As a measurement interval, two absorbance spectra and 12 oxygen data points per minute were collected, which provided the time resolution desired by physiologists. The mouse breathing with approximately $15 \mathrm{~mL} / \mathrm{min}$ resulted in a gas exchange time of $1.2 \mathrm{~s}$ within the iHWG.

Calibration. Calibration samples of ${ }^{13} \mathrm{CO}_{2},{ }^{12} \mathrm{CO}_{2}$, and oxygen

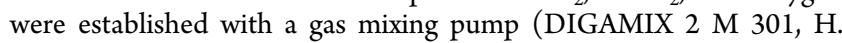
Wösthoff Messtechnik GmbH, Bochum, Germany) and using a static mixing method based on induced turbulences between $50 \mathrm{~mL}$ syringes (B. Braun Melsungen AG, Melsungen, Germany). All gas calibration samples were manufactured from pure technical grade nitrogen, pure technical grade carbon dioxide, and pure medical grade oxygen (all MTI Industriegase, Neu-Ulm, Germany). Certified test gases with known $\mathrm{CO}_{2}$ and $\mathrm{O}_{2}$ concentrations were used for validation purposes.

\section{ASSOCIATED CONTENT}

\section{S Supporting Information}

The Supporting Information is available free of charge on the ACS Publications website at DOI: 10.1021/acssensors.8b00477.

SEM image of an electrospun layer of polymer nanofibers, output power characteristic at a heat sink temperature of $15{ }^{\circ} \mathrm{C}$, wavelength tuning characteristic as a function of operating current and temperature, and response curve of the oxygen sensor based on electrospun nanofibers to four different oxygen concentrations (PDF)

\section{AUTHOR INFORMATION}

\section{Corresponding Author}

*E-mail: boris.mizaikoff@uni-ulm.de. Tel.: +49 731 50-22750.

\section{ORCID}

Boris Mizaikoff: 0000-0002-5583-7962

\section{Author Contributions}

E.T., M.N., and A.T. conceived the experiments. M.N was responsible for the system configuration and design. The midinfrared source was delivered by S.B., M.F., and J.K. The oxygen sensors were built by C.W., S.K., and V.R. Mouse breath experiments were performed by M.G., S.K., and M.W. Data interpretation was performed in cooperation with U.W., J.V., and P.R. The manuscript was written by E.T. and B.M. All 
authors discussed the results and contributed to the preparation of the manuscript.

\section{Notes}

The authors declare no competing financial interest.

\section{ACKNOWLEDGMENTS}

The authors acknowledge the support of the team operating the mouse intensive care unit (MICU) at the Institute of Anesthesiologic Pathophysiology and Method Development at the Ulm University Medical Center for assistance during the mouse breath studies. Partial support of this study by the project APOSEMA funded by the German BMBF within the M-Era.net program is greatly acknowledged. This work has been supported by EC's H2020 Program H2020-MSCA-RISE2014, Grant Agreement No. 645758 (“TROPSENSE”). The Machine Shop at Ulm University is thanked for support during prototype development of the dciHWG.

\section{ABBREVIATIONS}

dciHWG,dual-channel substrate-integrated hollow waveguide; DFB,distributed feedback; EBA,exhaled breath analysis; GCMS,gas chromatography mass spectrometry; ICL,interband cascade laser; ICU,intensive care unit; IRMS,isotope ratio mass spectrometry; LOD,limit of detection; RQ,respiratory quotient; TDLAS,tunable diode laser absorption spectroscopy,

\section{REFERENCES}

(1) Atherton, J. C.; Spiller, R. C. The Urea Breath Test for Helicobacter Pylori. Gut 1994, 35 (6), 723-725.

(2) Goddard, A. F.; Logan, R. P. H. Review Article: Urea Breath Tests for Detecting Helicobacter Pylori. Aliment. Pharmacol. Ther. 1997, 11 (4), 641-649.

(3) Schnabel, R.; Fijten, R.; Smolinska, A.; Dallinga, J.; Boumans, M.-L.; Stobberingh, E.; Boots, A.; Roekaerts, P.; Bergmans, D.; van Schooten, F. J. Analysis of Volatile Organic Compounds in Exhaled Breath to Diagnose Ventilator-Associated Pneumonia. Sci. Rep. 2015, 5, 17179.

(4) Vandenvelde, S.; Nevens, F.; Vanhee, P.; Vansteenberghe, D.; Quirynen, M. GC-MS Analysis of Breath Odor Compounds in Liver Patients. J. Chromatogr. B: Anal. Technol. Biomed. Life Sci. 2008, 875 (2), 344-348.

(5) Barth, E.; Tugtekin, I.; Weidenbach, H.; Wachter, U.; Vogt, J.; Radermacher, P.; Adler, G.; Georgieff, M. Determination of 13CO2/ $12 \mathrm{CO} 2$ Ratio by IRMS and NDIRS. Isot. Environ. Health Stud. 1998, 34 (1-2), 209-213.

(6) Hofstetter, D.; Beck, M.; Faist, J.; Nägele, M.; Sigrist, M. W. Photoacoustic Spectroscopy with Quantum Cascade DistributedFeedback Lasers. Opt. Lett. 2001, 26 (12), 887.

(7) Neri, G.; Lacquaniti, A.; Rizzo, G.; Donato, N.; Latino, M.; Buemi, M. Real-Time Monitoring of Breath Ammonia during Haemodialysis: Use of Ion Mobility Spectrometry (IMS) and Cavity Ring-down Spectroscopy (CRDS) Techniques. Nephrol., Dial., Transplant. 2012, 27 (7), 2945-2952.

(8) Dallner, M.; Scheuermann, J.; Nähle, L.; Fischer, M.; Koeth, J.; Höfling, S.; Kamp, M. InAs-Based Distributed Feedback Interband Cascade Lasers. Appl. Phys. Lett. 2015, 107 (18), 181105.

(9) von Edlinger, M.; Scheuermann, J.; Weih, R.; Zimmermann, C.; Nähle, L.; Fischer, M.; Koeth, J.; Höfling, S.; Kamp, M. Monomode Interband Cascade Lasers at $5.2 \$ \backslash \mathrm{mu} \backslash \mathrm{rm} \mathrm{M} \$$ for Nitric Oxide Sensing. IEEE Photonics Technol. Lett. 2014, 26 (5), 480-482.

(10) Scheuermann, J.; Weih, R.; von Edlinger, M.; Nähle, L.; Fischer, M.; Koeth, J.; Kamp, M.; Höfling, S. Single-Mode Interband Cascade Lasers Emitting below 2.8 Mm. Appl. Phys. Lett. 2015, 106 (16), 161103.

(11) Tütüncü, E.; Nägele, M.; Fuchs, P.; Fischer, M.; Mizaikoff, B. iHWG-ICL: Methane Sensing with Substrate-Integrated Hollow
Waveguides Directly Coupled to Interband Cascade Lasers. ACS Sens. 2016, 1 (7), 847-851.

(12) José Gomes da Silva, I.; Tütüncü, E.; Nägele, M.; Fuchs, P.; Fischer, M.; Raimundo, I. M.; Mizaikoff, B. Sensing Hydrocarbons with Interband Cascade Lasers and Substrate-Integrated Hollow Waveguides. Analyst 2016, 141, 4432-4437.

(13) Wolf, C.; Tscherner, M.; Köstler, S.; Ribitsch, V. Optochemical Sensors Based on Polymer Nanofibers with Ultra-Fast Response Characteristics. IEEE SENSORS 2014 Proceedings 2014, 950-953.

(14) Gordon, I. E.; Rothman, L. S.; Hill, C.; Kochanov, R. V.; Tan, Y.; Bernath, P. F.; Birk, M.; Boudon, V.; Campargue, A.; Chance, K. V. The HITRAN2016 Molecular Spectroscopic Database. J. Quant. Spectrosc. Radiat. Transfer 2017, 203, 3.

(15) Allan, D. W. Statistics of Atomic Frequency Standards. Proc. IEEE 1966, 54 (2), 221-230.

(16) Werle, P. O.; Mücke, R.; Slemr, F. The Limits of Signal Averaging in Atmospheric Trace-Gas Monitoring by Tunable DiodeLaser Absorption Spectroscopy (TDLAS). Appl. Phys. B: Photophys. Laser Chem. 1993, 57 (2), 131-139.

(17) Wilk, A.; Seichter, F.; Kim, S.-S.; Tütüncü, E.; Mizaikoff, B.; Vogt, J. A.; Wachter, U.; Radermacher, P. Toward the Quantification of the $13 \mathrm{CO} 2 / 12 \mathrm{CO} 2$ Ratio in Exhaled Mouse Breath with MidInfrared Hollow Waveguide Gas Sensors. Anal. Bioanal. Chem. 2012, 402 (1), 397-404.

(18) Wörle, K.; Seichter, F.; Wilk, A.; Armacost, C.; Day, T.; Godejohann, M.; Wachter, U.; Vogt, J.; Radermacher, P.; Mizaikoff, B. Breath Analysis with Broadly Tunable Quantum Cascade Lasers. Anal. Chem. 2013, 85 (5), 2697-2702.

(19) Seichter, F.; Wilk, A.; Wörle, K.; Kim, S.-S.; Vogt, J. A.; Wachter, U.; Radermacher, P.; Mizaikoff, B. Multivariate Determination of $13 \mathrm{CO} 2 / 12 \mathrm{CO} 2$ Ratios in Exhaled Mouse Breath with MidInfrared Hollow Waveguide Gas Sensors. Anal. Bioanal. Chem. 2013, 405 (14), 4945-4951.

(20) Halter, J. M.; Steinberg, J. M.; Schiller, H. J.; DaSilva, M.; Gatto, L. A.; Landas, S.; Nieman, G. F. Positive End-Expiratory Pressure after a Recruitment Maneuver Prevents Both Alveolar Collapse and Recruitment/Derecruitment. Am. J. Respir. Crit. Care Med. 2003, 167 (12), 1620-1626.

(21) Reiss, L. K.; Kowallik, A.; Uhlig, S. Recurrent Recruitment Manoeuvres Improve Lung Mechanics and Minimize Lung Injury during Mechanical Ventilation of Healthy Mice. PLoS One 2011, 6 (9), e24527.

(22) Wagner, F.; Wagner, K.; Weber, S.; Stahl, B.; Knöferl, M. W.; Huber-Lang, M.; Seitz, D. H.; Asfar, P.; Calzia, E.; Senftleben, U.; et al. Inflammatory Effects of Hypothermia and Inhaled H2S During Resuscitated, Hyperdynamic Murine Septic Shock. Shock 2011, 35 (4), 396-402. 